\title{
Adrenal metastasis of anaplastic meningioma: report of a rare case
}

\author{
Jiri Soukup ${ }^{1}$, Petra Kasparova ${ }^{1}$, Milan Vajda ${ }^{2}$, Ales Ryska ${ }^{1}$ \\ ${ }^{1}$ The Fingerland Department of Pathology, Charles University, Faculty of Medicine and University Hospital in Hradec Kralove, Czech \\ Republic, ${ }^{2}$ Department of Radiology, Charles University, Faculty of Medicine and University Hospital in Hradec Kralove, Czech Republic
}

\begin{abstract}
Meningiomas are the most common primary extra-axial tumours of the central nervous system, however their metastatic spread beyond central nervous system is rare. Here we present the case of a 54-year-old male with anaplastic meningioma who, 1.5 years after initial diagnosis, developed a tumorous expansion in his left adrenal gland, showing octreotide uptake on scintigraphy. Clinical diagnosis of pheochromocytoma was made and left adrenalectomy performed. The tumour weighed 480 grams and measured up to $140 \mathrm{~mm}$ in diameter. On histologic examination, morphology consistent with the diagnosis of anaplastic meningioma was present, resembling the original central nervous system tumour. The tumour expressed strongly SSTR2A and focally epithelial membrane antigen, $p 63$ and pancytokeratin (AE1/3). An extensive panel of neuronal and additional epithelial markers (SOX10, synaptophysin, chromogranin, inhibin, calretinin, BER-EP4, MOC31) was negative. The review of the literature on meningioma metastasising outside the central nervous system and on its differential diagnosis is provided.
\end{abstract}

Key words: metastasis, meningioma, rare tumours.

\section{Introduction}

Meningiomas are the most common primary extra-axial tumours of the central nervous system (CNS). They are usually confined to the CNS or show only local spread beyond its boundaries. Metastasizing meningiomas are rare and can be seen in atypical (WHO grade II) and anaplastic (WHO grade III) tumours [1-3,9,15]. Primary extracranial meningiomas are rare [13], however, metastasis or direct spread from the CNS lesion must be excluded before such diagnosis is made. Here, we present the case of anaplastic meningioma metastasising to the adrenal gland in a 54-year-old male, simulating pheochromocytoma clinically.

\section{Material and methods}

In January 2015, a 52-year-old male underwent a resection of an atypical meningioma localised in the right frontal area. Recurrent tumour was again resected in October 2015 (Fig. 1A), fulfilling criteria for anaplastic meningioma (34 mitoses/10 HPF (high power fields), focal areas of sarcomatoid appearance, Fig. 2B). The patient received radiotherapy (total 60 Gy in 30 fractions) in December 2015/January 2016. In November 2016, another surgery was performed due to the tumour recurrence (Fig. 1B, Fig. 2C, D) and the patient received treatment with Leksell gamma knife. During scintigraphy with octreotide performed for meningioma restaging in May 2017 

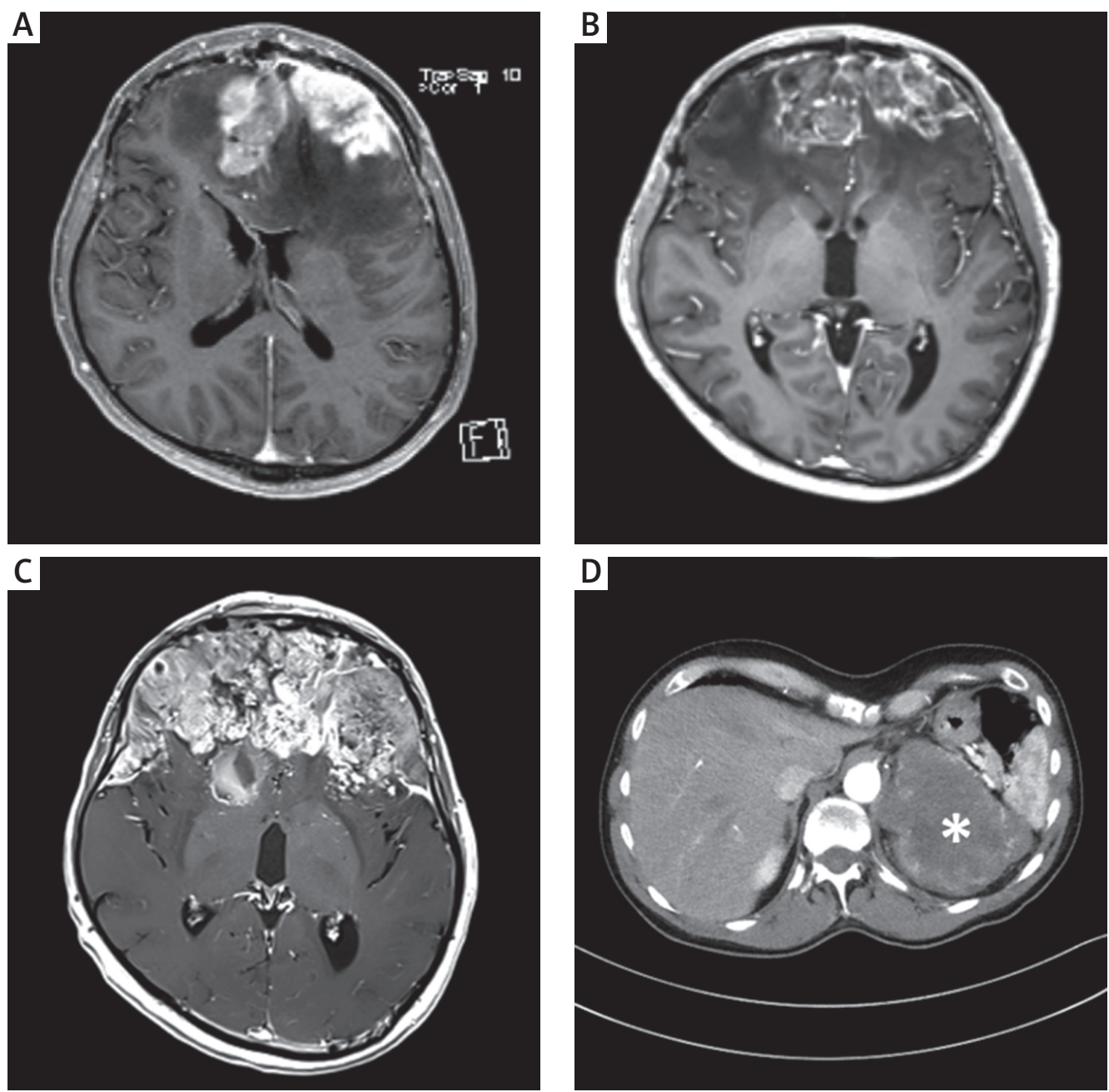

Fig. 1. Radiologic findings: first tumour recurrence in the frontal area, October 2015, T1 MRI (A); second tumour recurrence, November 2016, T1 MRI (B); third tumour recurrence, September 2017, T1 MRI (C); tumour of the adrenal gland (asterisk), May 2017, contrast-enhanced CT scan (D).

a $110 \mathrm{~mm}$ large mass in the left adrenal gland was discovered, showing octreotide uptake. The mass showed an expansive growth and a central necrosis on computed tomography (CT) scan - features radiologically suggestive of pheochromocytoma (Fig. 1D, asterisk). Clinical symptoms of catecholamine overproduction, however, were not observed. Based on the radiological findings, clinical diagnosis of pheochromocytoma was made and in June 2017, adrenalectomy was performed. The specimen consisted of a well-demarcated tumour with remnants of adrenal tissue. The tumour measured $140 \times 110$ $\times 75 \mathrm{~mm}$ and weighed $480 \mathrm{~g}$. The tissue was fixed in formalin and embedded in paraffin. Four $\mu \mathrm{m}$ thick sections were cut for haematoxylin and eosin (H\&E) staining and additional immunohistochemical studies. Multiple antibodies were used for diagnosis (Table I). The staining was conducted on the Benchmark Ultra stainer from Ventana/Roche with either ultraView Universal DAB detection kit or Optiview DAB IHC detection kit, using avidin-biotin complex method with horseradish peroxidase as an enzyme and 3,3'-diaminobenzidine for a substrate. For the antibodies against WT1, calretinin, CD57 and CD99, antigen retrieval was performed in a water bath for 40 min at $97^{\circ} \mathrm{C}$ at pH 9 (buffer Flex, Dako, Glostrup, Denmark). Endogenous peroxidase activity was inhibited by $3 \%$ hydrogen peroxide. After incubation with the antibody, the sections were subjected to EnVisionTM FLEX (Dako, lostrup, Denmark) visualization system. Slides were counterstained with haematoxylin. 

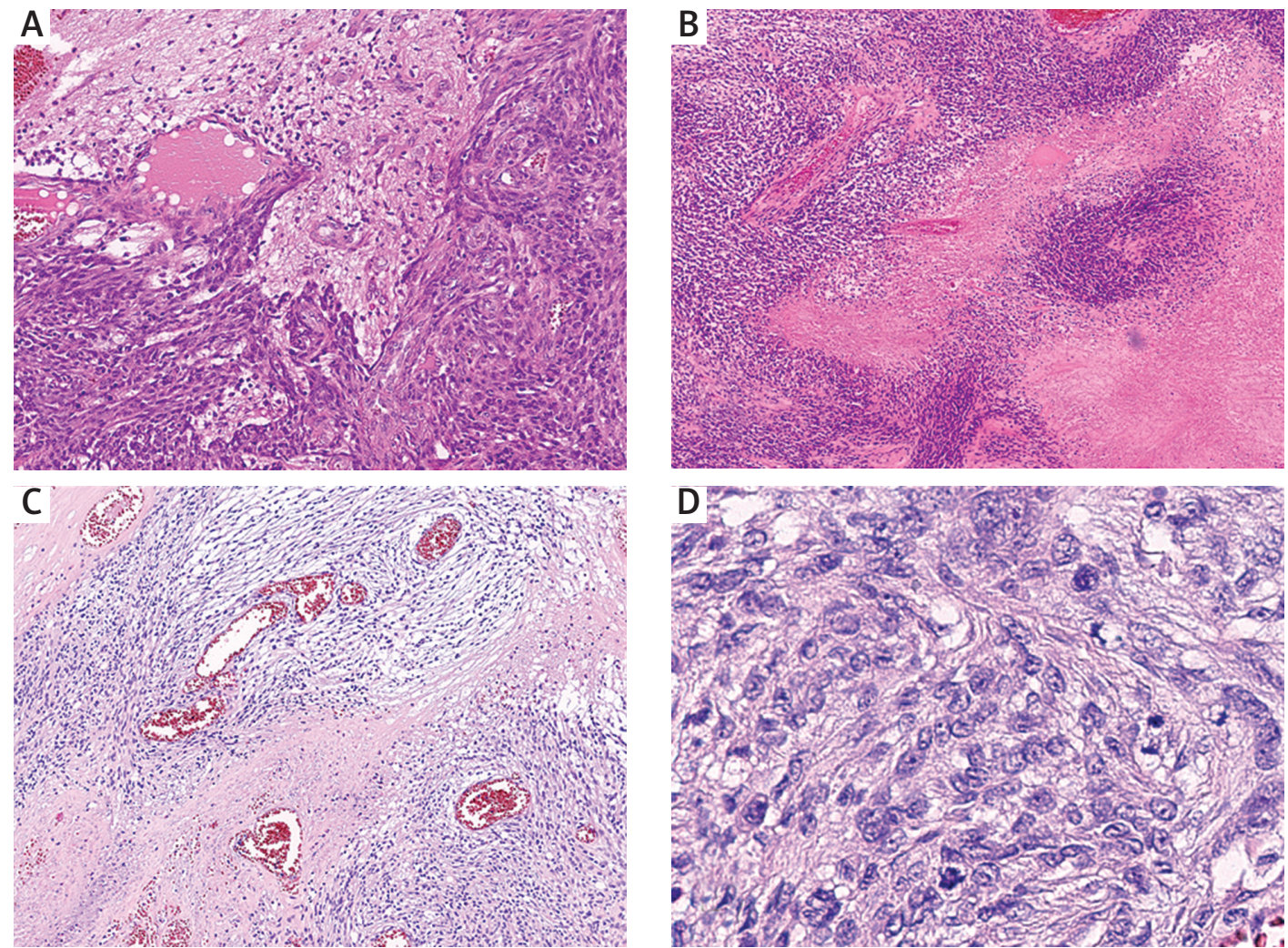

Fig. 2. Areas of brain invasion in atypical meningioma, January 2015 (A); geographic necrosis in anaplastic meningioma, October 2015 (B); anaplastic meningioma, November 2016 (C); mitotic activity in anaplastic meningioma, November 2016 (D).

The patient developed subsequently another recurrence of the primary tumour (September 2017, Fig. 1C) and a palliative approach was chosen. No further data about the patient were available at our institution.

\section{Results}

The tumour was encapsulated and composed of slender spindled cells arranged in long intersecting fascicles, in a storiform pattern or in vague whorls. Hypercellular areas alternated with collagenised regions (Fig. 3). There were areas of sharply demarcated coagulative necrosis (Fig. 4). Cells were relatively uniform with slightly eosinophilic cytoplasm, showing no significant cytological atypias (Fig. 5). In multiple areas, the cells formed small onionskin-like structures (Fig. 6). Mitotic activity reached up to 26 mitoses/10 HPF. The overall morphology resembled the anaplastic meningiomas diagnosed in October 2015 and November 2016 (Fig. 2).

The tumour stained strongly and diffusely for SSTR2A and vimentin. Pancytokeratin and EMA anti- bodies showed focal positivity (Fig. 7). Weak focal positivity of p63 was also observed. All other tested markers (synaptophysin, chromogranin, S-100 protein, SOX10, CD56, CD57, GFAP, melan A, HMB45, inhibin, CD34, CD99, progesterone receptors, calretinin, desmin, cytokeratin 7, WT1, BER-EP4, MOC31) were negative. Ki67 was positive in approximately $30 \%$ of tumour cells. A diagnosis of metastatic anaplastic meningioma was established.

\section{Discussion}

Meningiomas are the most common extra-axial neoplasms, accounting for almost $35 \%$ of the primary CNS tumours. Most commonly, they arise from the meninges, although rare intraventricular cases exist, arising from arachnothelial cells around choroid plexus [5]. The current WHO classification recognises 3 grades of meningioma, distinguished by morphologic features (Table II). Meningiomas commonly express epithelial membrane antigen (EMA) and progesterone receptors, although the extent 
Table I. Antibodies used

\begin{tabular}{|c|c|c|c|}
\hline Antibody & Clone & Manufacturer & Dilution \\
\hline Pancytokeratin & $\mathrm{AE} 1 / 3$ & Dako, Glostrup, Denmark A/S & $1: 75$ \\
\hline Cytokeratin 7 & OV-TL $12 / 30$ & Dako, Glostrup, Denmark A/S & $1: 100$ \\
\hline Epithelial membrane antigen & E29 & Dako, Glostrup, Denmark A/S & $1: 400$ \\
\hline Ki67 & $30-9$ & Ventana, Basel, Switzerland & prediluted \\
\hline Vimentin & V9 & Ventana, Basel, Switzerland & $1: 400$ \\
\hline SSTR2A & UMB1 & Abcam, Cambridge, MA, USA & $1: 1500$ \\
\hline Synaptophysin & SP11 & Ventana, Basel, Switzerland & prediluted \\
\hline Chromogranin & LK2H10 & Cell Marque Rocklin, CA, USA & $1: 1000$ \\
\hline Progesterone receptors & polyclonal & Ventana, Basel, Switzerland & prediluted \\
\hline BER-EP4 & BER-EP4 & Dako, Glostrup, Denmark A/S & $1: 400$ \\
\hline MOC31 & MOC31 & Dako, Glostrup, Denmark A/S & $1: 500$ \\
\hline WT1 & GF-HL & Dako, Glostrup, Denmark A/S & $1: 50$ \\
\hline GFAP & EP672Y & Ventana, Basel, Switzerland & prediluted \\
\hline $\mathrm{S} 100$ & polyclonal & Dako, Glostrup, Denmark A/S & $1: 5000$ \\
\hline SOX10 & EP268 & Cell Marque Rocklin, CA, USA & $1: 50$ \\
\hline Desmin & D33 & Dako, Glostrup, Denmark A/S & $1: 100$ \\
\hline Inhibin & alpha(R1) & Cell Marque Rocklin, CA, USA & $1: 25$ \\
\hline HMB45 & HMB45 & Dako, Glostrup, Denmark A/S & $1: 50$ \\
\hline Melan A & A103 & Dako, Glostrup, Denmark A/S & $1: 10$ \\
\hline CD34 & QBEnd 10 & Dako, Glostrup, Denmark A/S & $1: 50$ \\
\hline CD56 & MRQ-42 & Cell Marque Rocklin, CA, USA & $1: 2000$ \\
\hline CD57 & TB01 & Dako, Glostrup, Denmark A/S & $1: 50$ \\
\hline CD99 & $12 \mathrm{E} 7$ & Dako, Glostrup, Denmark A/S & $1: 50$ \\
\hline p63 & $4 \mathrm{~A} 4$ & Ventana, Basel, Switzerland & prediluted \\
\hline Calretinin & DAK-calret 1 & Dako, Glostrup, Denmark A/S & $1: 50$ \\
\hline
\end{tabular}

and intensity of staining may vary, being weak or even absent especially in high grade tumours. Somatostatin receptors 1-5 (SSTRs) are expressed on a regular basis [16], leading to uptake of somatostatin analogue octreotide which can be demonstrated on a scintigraphy. SSTR2A in particular is a sensitive marker of meningiomas, including anaplastic tumours, being expressed in $95 \%$ to $100 \%$ of cases, usually in a strong and diffuse fashion [7,8]. Another recently described sensitive marker of meningothelial differentiation is MUC4: its expression was described in almost $93 \%$ of cases [7]. Cytokeratins may be expressed in meningiomas, especially in the high grade cases, and CEA expression is a regular feature of the secretory variant of meningioma [4]. P63 is expressed in a subset of meningiomas, especially grade II and III [14].

Extracranial metastases of meningiomas are rare, but not unknown in the literature [1-3,9,15]. In a large report of 396 meningiomas, there were
Table II. WHO grading system of meningiomas [5]

\begin{tabular}{|lc|}
\hline $\begin{array}{l}\text { Atypical meningioma } \\
\text { (WHO grade II) }\end{array}$ & $\begin{array}{c}\text { Anaplastic meningioma } \\
\text { (WHO grade III) }\end{array}$ \\
\hline Brain invasion OR & $\begin{array}{c}\text { Obviously malignant } \\
\text { cytology OR }\end{array}$ \\
\hline $\begin{array}{l}\text { Mitotic count } \geq 4 \text { mitoses/ } \\
10 \text { HPF OR }\end{array}$ & $\begin{array}{l}\text { Mitotic count } \geq 20 \\
\text { mitoses/10 HPF OR }\end{array}$ \\
\hline $\begin{array}{l}\text { Three or more of following } \\
\text { features: small cell } \\
\text { component, hypercellularity, } \\
\text { necrosis, macronucleoli, loss } \\
\text { of growth pattern OR }\end{array}$ & $\begin{array}{l}\text { Special morphologic subtype } \\
\text { (either papillary or rhabdoid) }\end{array}$ \\
\hline $\begin{array}{l}\text { Special morphologic subtype } \\
\text { (either chordoid or clear cell) }\end{array}$ & \\
\hline
\end{tabular}

7 (1.76\%) anaplastic meningiomas, of which 3 (43\%) had metastasized [1]. Rarely, metastases are observed in atypical meningiomas [3] and also in grade I meningiomas [9]. The most common target organs are lungs, pleura, bones and liver. Meningiomas can 


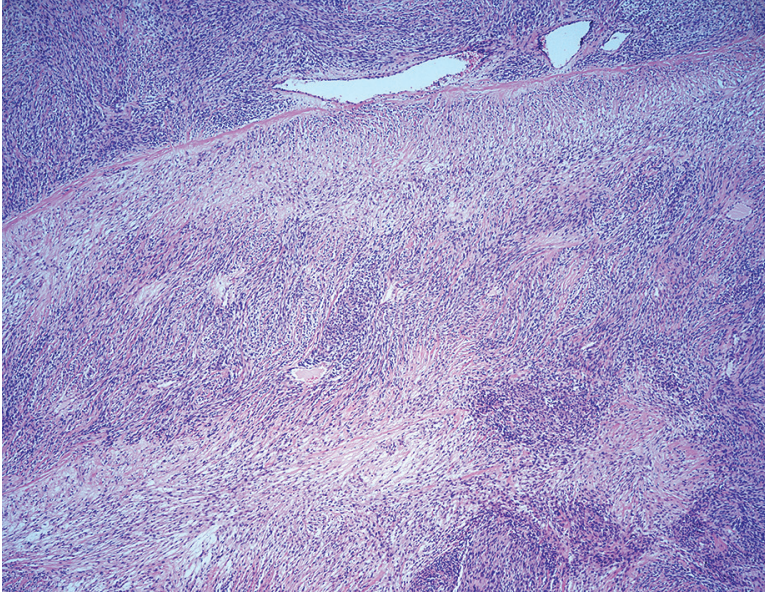

Fig. 3. Hypercellular areas alternating with more collagenised regions (H\&E).

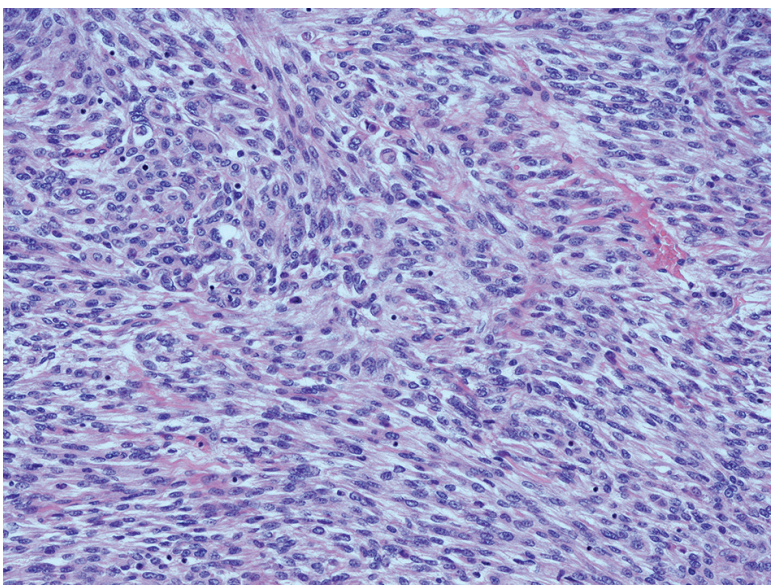

Fig. 5. Relatively uniform cells without significant atypia (H\&E).

invade veins and dural sinuses, reaching for systemic circulation. The leptomeningeal spread via cerebrospinal fluid is also possible, with the reported incidence of around $0.9 \%$ (5 out of 534 resected meningiomas) in another large series [12]; these included tumours of all grades. Older reports of metastasising meningiomas must be regarded with some scrutiny since they may include meningeal solitary fibrous tumours or other rarer meningeal tumours. This is presumably reflected by a higher proportion of metastatic "angioblastic" meningiomas in older reports [15].

Primary extracranial meningiomas do exist, however, they are rare outside the region of the head and neck [13]. Most commonly, they arise in the middle ear, nasal cavity, or skin of scalp and the sacrococ-

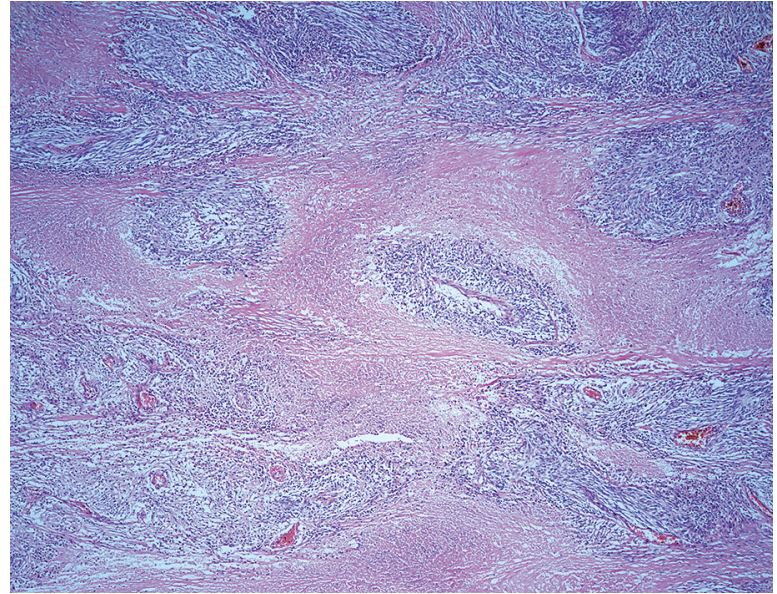

Fig. 4. Geographic areas of coagulative necrosis (H\&E).

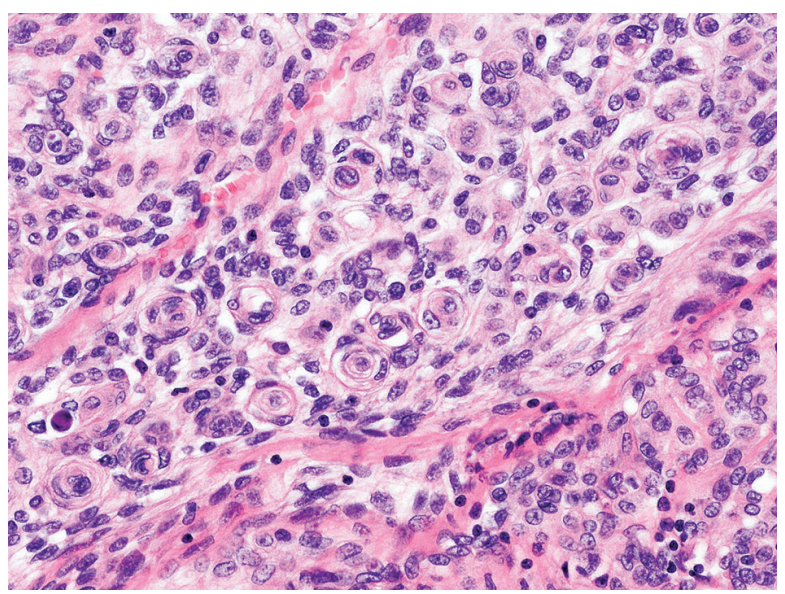

Fig. 6. Small onionskin-like structures scattered throughout the tumour (H\&E).

cygeal area. It is hypothesised that they originate from arachnoid cell wrappings around vessels and nerves in skull foramina, from displaced arachnoid cells due to the intracranial hypertension, from displacement of arachnoid cells during embryogenesis, or from undifferentiated precursor mesenchymal cells [13]. Most of the extracranial meningiomas are represented by the meningothelial variant, although other subtypes, including clear cell, atypical and anaplastic meningiomas may be found. The possibility of extracranial spread of a primary CNS neoplasm or a metastasis must be excluded before the diagnosis of primary extracranial meningioma is made. The immunohistochemical profile of extracranial meningiomas does not differ from their intracranial counterparts [13]. 

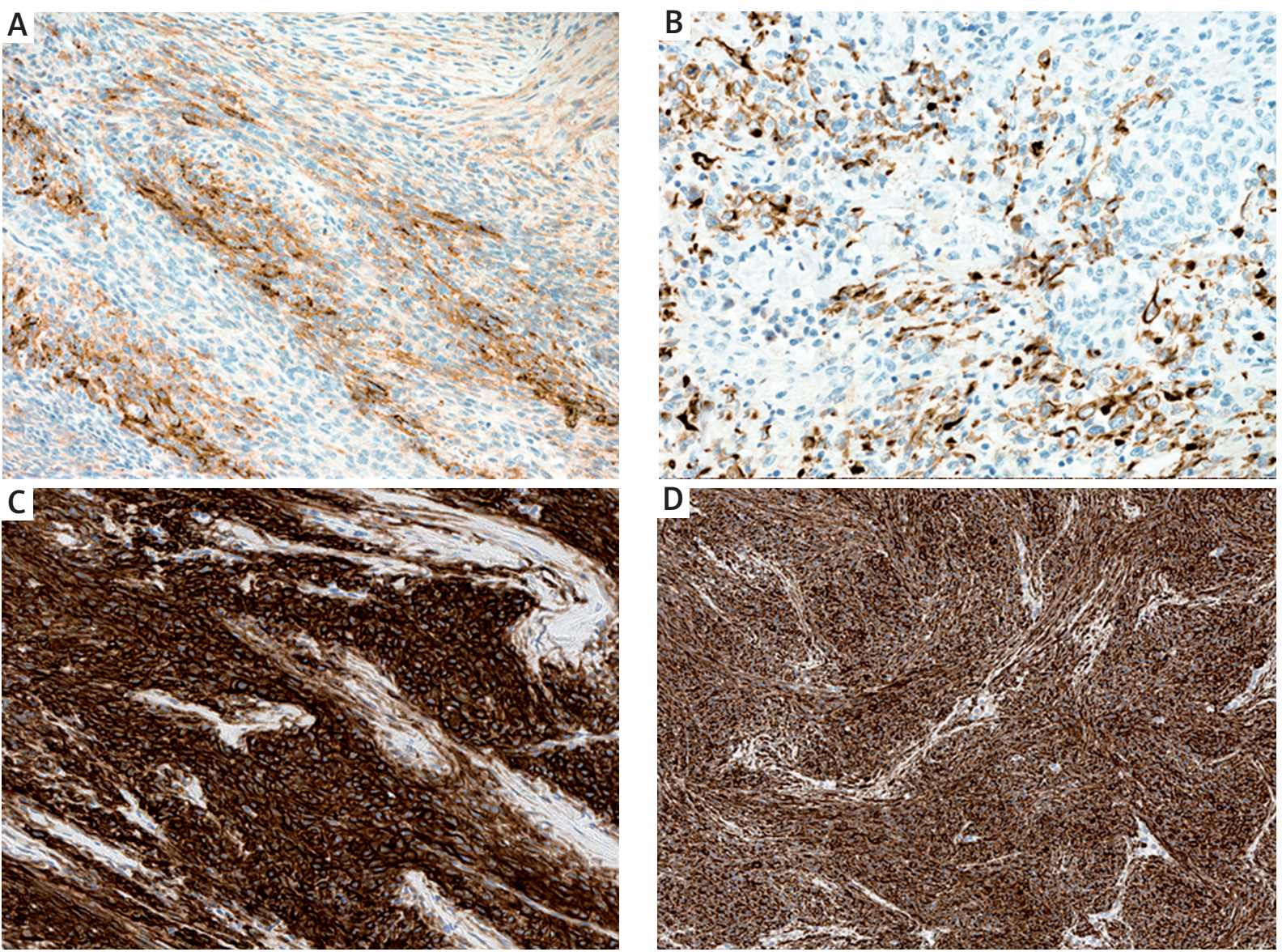

Fig. 7. EMA positivity of tumour cells (A), pancytokeratin (AE1/3) positivity of scattered cells (B), diffuse and strong positivity of SSTR2A (C), and vimentin (D).

Differential diagnosis in our case included several entities: sarcomatoid differentiation can be present in the adrenal cortical carcinoma, but such tumours are extremely rare $[6,17]$. Careful sampling usually allows at least focal identification of the epithelial component which expresses inhibin or melan A. Pheochromocytomas have different morphology and express synaptophysin and chromogranin. Cases of hybrid pheochromocytoma and malignant peripheral neural sheath tumour (MPNST) were described [10]. MPNST is a spindle cell sarcoma showing areas of increased cellularity alternating with myxoid, less cellular areas. There is usually weak and focal positivity of some "neural" markers like S100, SOX10, GFAP, or CD57. S100 positivity may be encountered in meningiomas but SOX10 shows much higher sensitivity for neurogenic differentiation and only rarely stains meningiomas [11]. Strong S100 and SOX10 positivity would therefore distinguish Schwanno- ma, ganglioneuroma and neurofibroma. In conclusion, extracranial metastases from the primary CNS meningiomas are rare but do occur, especially in atypical and anaplastic meningiomas. We have herein described such an unusual case of adrenal metastasis of anaplastic meningioma, with a short overview of the differential diagnosis.

\section{Acknowledgements and funding information}

This case report was supported from the European Regional Development Fund-Project BBMRI-CZ: Biobank network - a versatile platform for the research of the etiopathogenesis of diseases, No: EF16 013/0001674. The funding source had no involvement in the study design, collection, analysis, and interpretation of data, on the writing of the report, or on the decision to submit the article for publication. 


\section{Disclosure}

The authors report no conflict of interest.

\section{References}

1. Enam SA, Abdulrauf S, Mehta B, Malik GM, Mahmood A. Metastasis in meningioma. Acta Neurochir (Wien) 1996; 138: 1172 1177; discussion 1177-1178.

2. Karasick JL, Mullan SF. A survey of metastatic meningiomas. J Neurosurg 1974; 40: 206-212.

3. Lee GC, Choi SW, Kim SH, Kwon HJ. Multiple extracranial metastases of atypical meningiomas. J Korean Neurosurg Soc 2009; 45: 107-111.

4. Liu Y, Sturgis CD, Bunker M, Saad RS, Tung M, Raab SS, Silverman JF. Expression of cytokeratin by malignant meningiomas: diagnostic pitfall of cytokeratin to separate malignant meningiomas from metastatic carcinoma. Mod Pathol 2004; 17: 11291133.

5. Louis DN, Ohgaki H, Wiestler OD, Cavenee WK. (eds.). WHO classification of tumours of the central nervous system. Revised $4^{\text {th }}$ ed. IARC, Lyon 2016.

6. Mark D, Boyd C, Eatock F. Adrenal sarcomatoid carcinoma: a case report and review of the literature. Ulster Med J 2014; 83: 89-92.

7. Matsuyama A, Jotatsu M, Uchihashi K, Tsuda Y, Shiba E, Haratake J, Hisaoka M. MUC4 expression in meningiomas: under-recognized immunophenotype particularly in meningothelial and angiomatous subtypes. Histopathology 2019; 74: 276-283.

8. Menke JR, Raleigh DR, Gown AM, Thomas S, Perry A, Tihan T. Somatostatin receptor $2 a$ is a more sensitive diagnostic marker of meningioma than epithelial membrane antigen. Acta Neuropathol 2015; 130: 441-443.

9. Miller DC, Ojemann RG, Proppe KH, McGinnis BD, Grillo HC. Benign metastasizing meningioma. Case report. J Neurosurg 1985; 62: 763-766.

10. Namekawa T, Utsumi T, Imamoto T, Kawamura K, Oide T, Tanaka T, Nihei N, Suzuki H, Nakatani Y, Ichikawa T. Composite pheochromocytoma with a malignant peripheral nerve sheath tumor: Case report and review of the literature. Asian J Surg 2016; 39: 187-190.

11. 11. Ng J, Celebre A, Munoz DG, Keith JL, Karamchandani JR. Sox10 is superior to $\mathrm{S} 100$ in the diagnosis of meningioma. Appl Immunohistochem Mol Morphol 2015; 23: 215-219.

12. Park KS, Kim KH, Park SH, Hwang JH, Lee DH. Intracranial meningioma with leptomeningeal dissemination: retrospective study with review of the literature. J Korean Neurosurg Soc 2015; 57: 258-265.

13. Rushing EJ, Bouffard JP, McCall S, Olsen C, Mena H, Sandberg GD, Thompson LD. Primary extracranial meningiomas: an analysis of 146 cases. Head Neck Pathol 2009; 3: 116-130.

14. Rushing EJ, Olsen C, Man YG. Correlation of p63 immunoreactivity with tumor grade in meningiomas. Int J Surg Pathol 2008; 16: 38-42.

15. Shuangshoti S, Hongsaprabhas C, Netsky MG. Metastasizing meningioma. Cancer 1970; 26: 832-841.
16. Silva CB, Ongaratti BR, Trott G, Haag T, Ferreira NP, Leaes CG, Pereira-Lima JF, Oliveira Mda C. Expression of somatostatin receptors (SSTR1-SSTR5) in meningiomas and its clinicopathological significance. Int J Clin Exp Pathol 2015; 8: 13185-13192.

17. Sturm N, Moulai N, Laverriere MH, Chabre O, Descotes JL, Brambilla E. Primary adrenocortical sarcomatoid carcinoma: case report and review of literature. Virchows Arch 2008; 452: 215219. 